\title{
DEVELOPMENT AND EVALUATION OF FLAVOXATE HCI DOUBLE CORE COMPRESSED TABLET FORMULATIONS BY SWELLABLE GRANULATION TECHNIQUE
}

\author{
AHMAD JUNAID RASHID ${ }^{1,2}$, SAJID BASHIR ${ }^{1}$, QAZI AMIR IJAZ ${ }^{2}$, NASIR ABBAS ${ }^{2 *}$, \\ NADEEM IRFAN BUKHARI ${ }^{2}$, SAIQA ISHTIAQ ${ }^{2}$, SHAHID RASOOL ${ }^{1}$, ATIF RAZA $^{2}$, \\ and UBAID ULLAH ANWER ${ }^{2}$
}

${ }^{1}$ Faculty of Pharmacy College of Pharmacy, University of Sargodha, Sargodha, Pakistan

${ }^{2}$ Punjab University College of Pharmacy, University of the Punjab, Lahore, Pakistan

\begin{abstract}
The objective of the proposed research was to develop double core differential release tablets of Flavoxate $\mathrm{HCl}$, a smooth-muscle relaxant through the swellable technique. Inner core granules were prepared by a wet granulation method using HPMC K15 and Kollidon as sustained-release polymers. Outer core immediate release granules were also prepared by a wet granulation method using standard tableting excipients. Both types of granules were evaluated for pharmaceutical, structural, and thermal parameters. The double core tablet was prepared using a pre-compressed inner tablet. In vitro release studies were performed in $0.1 \mathrm{~N} \mathrm{HCl}(\mathrm{pH} 1.2)$ and phosphate buffer $(\mathrm{pH} 7.4)$ as dissolution media. Stability studies were also performed on the selected formulations. Pharmaceutical evaluation results of inner core granules prepared by HPMC K15 and Kollidon and tablets showed acceptable values for all the physical parameters. FTIR studies showed that the principal peaks of the drug remained intact, indicating no chemical interaction occurred between drug and a polymer. DSC results showed no noteworthy change in the thermal behavior of the drug while in granular formulation. Inner core tablets were successfully compressed with acceptable results of friability, hardness, thickness, diameter, and content uniformity parameters. Double core tablets were prepared by employing immediate release granules as the outer core with intact tablets as the inner core, and all the uniformity parameters of the prepared tablets were found to be within the acceptable limits. In vitro release studies in both media showed a characteristic sustained drug release pattern for up to 24 hours. Results of stability studies indicated that all the formulations remained stable.
\end{abstract}

Keywords: Flavoxate, swellable, double core, HPMC K15, Kollidon, polymer

Swellable matrices are either homogeneous or heterogeneous monolithic systems that are specifically used for the drugs that require frequent dosing. The polymers employed in these systems usually swell to form hydrogels when in contact with water (1). In swellable granulation, the drug delivery primarily depends on the outer gel layer, formed on the matrix surface because of polymer transition in dissolution media. This technique can also be helpful to prolong the drug delivery from the drug product because of the continuous swelling of polymeric substances. The drug release from the dosage form is mainly dependent on the interaction among the drug, polymer, and water $(2,3)$.

Hydroxypropyl methylcellulose (HPMC K15) is a biocompatible, swellable hydrophilic polymer, which is widely used for the controlled delivery of drugs from the dosage form (4). Kollidon SR is a $\mathrm{pH}$-independent sustained release polymer, composed of $80 \%$ Polyvinyl acetate and $19 \%$ povidone in a physical mixture, stabilized with $0.8 \%$ sodium lauryl sulfate and $0.2 \%$ colloidal silica. It is insoluble in water and very soluble in N-methylpyrrolidone.

Flavoxate, a smooth-muscle relaxant, works by relaxing the muscles in the bladder. It helps to prevent more frequent micturition and bladder pain. Its normal adult dose is $100-200 \mathrm{mg}$ per oral 3 to 4 times daily. It is normally excreted via kidneys with 10 $30 \%$ of the dose being excreted in urine within $6 \mathrm{~h}$ of dosing and $57 \%$ being excreted within $24 \mathrm{~h}$ of dosing (5). Its metabolite 3-methyl-flavone-8-carboxylic acid (MFCA) appears in urine both as free and conjugated

*Corresponding author: e-mail: nasir.pharmacy@pu.edu.pk; saiqa.pharmacy@pu.edu.pk 
form (6). The absolute bioavailability of the drug is $90 \%$. All these properties make Flavoxate HCI an ideal candidate for the development of a sustainedrelease formulation to reduce the frequency of dosing. Therefore, the present study was carried out to develop a Flavoxate double core tablet formulation containing an immediate-release layer for attending the fast onset of action and sustained release core using a swellable matrix technique with HPMC K15 and Kollidon SR as polymers and Povidone $\mathrm{K} 30$ as a binder (7-9). The swellable matrix formulations have been reported to have many advantages such as reduction in the size of a tablet as compared to hot melt granulation technique, better control over the drug release, and lesser side effects (10). In this study, nine batches each of HPMC K15 and Kollidon SR were prepared separately and evaluated for pharmaceutical properties.

\section{EXPERIMENTAL}

\section{Materials}

Flavoxate HCI, HPMC K15, Kollidon SR, and Povidone K30 were gifted by Pacific Pharmaceuticals (Lahore, Pakistan). All the other chemicals, i.e., Citric acid, Magnesium stearate, and Aerosil were obtained from Merck, Germany, while Avicel was obtained from Mingthai, Taiwan.

\section{Manufacturing of double core tablets}

Double core tablets containing sustained-releaseinner core granules and immediate release outer core were manufactured by the following methods.

\section{Preparation of inner core granules}

Nine batches of Flavoxate HCI inner core granules were manufactured by using different quantities of HPMC K15 and Kollidon SR as shown in Table 1. The weighed quantity of the binder was mixed with distilled water to make the paste. Weighed quantity of Flavoxate HCI and polymer (HPMC K15 and Kollidon SR) was passed from sieve No. 16 and mixed geometrically. Afterward, the binder paste was gradually added into the mixer and further mixed for $\sim 2 \mathrm{~min}$. The wet mass was dried at $50 \pm 5^{\circ} \mathrm{C}$ for $3 \mathrm{~h}$ and was converted into granules by using an automatic Granulator (Sieve No. 12). The granules were then mixed with magnesium stearate and citric acid for $10 \mathrm{~min}$ (11). To evaluate the properties of granules various quality control tests, i.e., sieve analysis, bulk and tapped density, loss on drying (LOD), and content uniformity test were performed according to the standard procedures (12).

Table 1. Composition of Flavoxate $\mathrm{HCl}$ sustained release inner core with HPMC K15 and Kollidon SR.

\begin{tabular}{|c|c|c|c|c|c|c|c|}
\hline Formulation & $\begin{array}{l}\text { HPMC } \\
\text { K15 }\end{array}$ & Citric acid & $\begin{array}{l}\text { Povidone } \\
\text { K30 (mg) }\end{array}$ & $\begin{array}{l}\text { Magnesium } \\
\text { stearate (mg) }\end{array}$ & $\begin{array}{l}\text { Kollidon SR } \\
\text { (mg) }\end{array}$ & $\begin{array}{c}\text { Kollidon SR } \\
\text { for paste }(\mathrm{mg})\end{array}$ & $\begin{array}{l}\text { Wt. of tablet } \\
\text { (mg) }\end{array}$ \\
\hline $\mathrm{ICG}_{\mathrm{H}-\mathrm{WG}}-\mathrm{I}$ & 50 & 10 & 20 & 10 & - & - & 590 \\
\hline $\mathrm{ICG}_{\mathrm{K}-\mathrm{WG}}-\mathrm{I}$ & - & - & - & 5 & 50 & 10 & 590 \\
\hline $\mathrm{ICG}_{\mathrm{H}-\mathrm{WG}}-\mathrm{II}$ & 65 & 10 & 25 & 10 & - & - & 610 \\
\hline $\mathrm{ICG}_{\mathrm{k}-\mathrm{WG}}-\mathrm{II}$ & - & - & - & 5 & 55 & 15 & 590 \\
\hline $\mathrm{ICG}_{\mathrm{H}-\mathrm{WG}}-\mathrm{III}$ & 90 & 10 & 30 & 10 & - & - & 640 \\
\hline $\mathrm{ICG}_{\mathrm{k}-\mathrm{WG}}$-III & - & - & - & 5 & 45 & 20 & 590 \\
\hline $\mathrm{ICG}_{\mathrm{H}-\mathrm{WG}} \mathrm{IV}$ & 100 & 10 & 30 & 10 & - & - & 650 \\
\hline $\mathrm{ICG}_{\mathrm{k}-\mathrm{WG}}-\mathrm{IV}$ & - & - & - & 5 & 50 & 15 & 590 \\
\hline $\mathrm{ICG}_{\mathrm{H}-\mathrm{WG}}-\mathrm{V}$ & 100 & 15 & 30 & 10 & - & - & 655 \\
\hline $\mathrm{ICG}_{\mathrm{k}-\mathrm{WG}}-\mathrm{V}$ & - & - & - & 5 & 50 & 15 & 590 \\
\hline $\mathrm{ICG}_{\mathrm{H}-\mathrm{WG}}-\mathrm{VI}$ & 100 & 20 & 30 & 10 & - & - & 660 \\
\hline $\mathrm{ICG}_{\mathrm{k}-\mathrm{WG}}-\mathrm{VI}$ & - & - & - & 5 & 50 & 10 & 590 \\
\hline $\mathrm{ICG}_{\mathrm{H}-\mathrm{WG}}-\mathrm{VII}$ & 100 & 10 & 30 & 10 & - & - & 650 \\
\hline $\mathrm{ICG}_{\mathrm{k}-\mathrm{WG}}-\mathrm{VII}$ & - & - & - & 5 & 50 & 15 & 590 \\
\hline $\mathrm{ICG}_{\mathrm{H}-\mathrm{WG}}-\mathrm{VIII}$ & 100 & 15 & 30 & 10 & - & - & 655 \\
\hline $\mathrm{ICG}_{\mathrm{k}-\mathrm{WG}}-\mathrm{VIII}$ & - & - & - & 5 & 55 & 20 & 590 \\
\hline $\mathrm{ICG}_{\mathrm{H}-\mathrm{WG}}-\mathrm{XI}$ & 100 & 20 & 30 & 10 & - & - & 660 \\
\hline $\mathrm{ICG}_{\mathrm{k}-\mathrm{WG}}-\mathrm{XI}$ & - & - & - & 5 & 55 & 15 & 590 \\
\hline
\end{tabular}

*H-WG stands for formulation prepared by wet granulation using HPMC K15\& k-WG for formulation prepared by wet granulation using kollidon SR 


\section{Preparation of outer core granules}

Three different immediate release granule batches of Flavoxate $\mathrm{HCl}$ were prepared using the wet granulation approach for the outer core, labeled as $\mathrm{OCG}_{\mathrm{WG}}$-I to $\mathrm{OCG}_{\mathrm{WG}}$-III (Table 2). Each outer core granules formulation was prepared by blending Flavoxate HCI (100 mg) and Avicel PH 101 as given in Table 2 (13). Both ingredients were sieved and mixed uniformly in a lab-scale mixer (Khan engineering, Lahore, Pakistan). The weighed quantity of Povidone $\mathrm{K} 30$ was mixed with $1 \mathrm{~L}$ of the distilled water. In order to form a smooth paste, the resultant solution was heated to $60^{\circ} \mathrm{C}$ to completely dissolve the Povidone $\mathrm{K} 30$. The paste was added gradually into the mixer of Flavoxate HCI and Avicel PH 101 and was mixed at slow speed for about $2 \mathrm{~min}$ (14). The granules were subjected to various tests, including physical appearance, sieve analysis, density studies, loss on drying, DSC, FTIR analysis, and content uniformity using the validate HPLC method described elsewhere.

\section{Differential calorimetry scanning (DSC)}

Differential scanning calorimeter (DSC) was used for thermal analysis of Flavoxate $\mathrm{HCl}$ granules for inner as well as outer core. The test was carried out with $5.0 \mathrm{mg}$ of Flavoxate $\mathrm{HCl}$ granules. These granules were sealed in a $30 \mu \mathrm{L}$ aluminum pan and scanned at $10-300^{\circ} \mathrm{C}$ at a heating rate of $10^{\circ} \mathrm{C} / \min (15)$.

\section{FTIR spectroscopy}

Fourier Transform Infrared (FTIR) spectra were recorded for the pure drug, inner core, and outer core granules by using an FTIR spectrophotometer (Alpha-P Bruker, Germany), equipped with an ATR unit across the range of $4000-400 \mathrm{~cm}^{-1}$

\section{Compression of sustained-release inner core tablets}

All the inner core granules formulations prepared by using different polymers (Table 1), were compressed into tablets using a rotary compression machine (Killian RUD, Germany) having 20 stations capable to make double core tables. It is a specialized machine capable of making double core tablets. The machine is equipped with two different hoppers, one is meant for pre-compressed tablets used as the inner core, whereas the other hopper is meant for the transfer of outer core granules to make a tablet within the tablet (double core tablets). The inner core was compressed using round, convex punches having a diameter of $12 \mathrm{~mm}$. The inner cores tablets were subjected to physical tests, including appearance, diameter, friability, hardness, thickness, and weight variation according to official British Pharmacopeia (BP) methods (12).

\section{Compression of double core tablets}

Outer core granules were filled in the hopper and its flow was adjusted. Already compressed inner core tablets were placed in the lower hopper. The speed of the tablet sonicator was adjusted to make a uniform flow of inner core tablets through the PVC tube of the tablet machine leading into the turret. The weight and thickness of the double core were adjusted as the preset limits. Once the parameters are achieved, the compressed double core tablets were subjected to below mentioned tests.

\section{Characterization of Flavoxate HCl double core tablets}

The compressed double core tablets were subjected to various physical and chemical quality control tests including appearance, diameter, hardness, thickness, weight variation, disintegration, and dissolution by standard procedures given in British Pharmacopeia (12).

\section{In vitro dissolution studies}

In-vitro release performance of the drug was determined by using the USP dissolution apparatus-II (Agilent, 708-DS, Germany). The dissolution medium $(900 \mathrm{~mL})$ was stirred at $100 \mathrm{rpm}$ and the temperature was maintained at $37 \pm 0.5^{\circ} \mathrm{C}$. The dissolution of double core tablets was performed in $0.1 \mathrm{~N} \mathrm{HCl}$ for $2 \mathrm{~h}$ and then in phosphate buffer $\mathrm{pH} 7.4$ for further

Table 2. Composition of outer core batches with Povidone K-30.

\begin{tabular}{|c|c|c|c|}
\hline Formulation composition & $\mathrm{OCG}_{\mathrm{WG}}-\mathrm{I}$ & $\mathrm{OCG}_{\mathrm{WG}}-\mathrm{II}$ & $\mathrm{OCG}_{\mathrm{WG}}-\mathrm{III}$ \\
\hline Flavoxate $\mathrm{HCl}(\mathrm{mg})$ & 100 & 100 & 100 \\
\hline Povidone K-30 (mg) & 9 & 15 & 20 \\
\hline Avicel PH 101 (mg) & 283 & 277 & 4 \\
\hline Magnesium Stearate (mg) & 4 & 4 & 4 \\
\hline Aerosil (mg) & 4 & 4 & 400 \\
\hline Total weight $(\mathrm{mg})$ & 400 & 400 & \\
\hline
\end{tabular}


$22 \mathrm{~h}$ (total time $2+22 \mathrm{~h}=24 \mathrm{~h}$ ). The sample was withdrawn initially at $30 \mathrm{~min}$ and then after 1 and $2 \mathrm{~h}$ with the help of auto sampler. The solution was filtered using a $0.22 \mu \mathrm{m}$ membrane filter and $5 \mathrm{ml}$ of this solution was transferred to a $100 \mathrm{ml}$ volumetric flask. The absorbance of the solution was measured at a wavelength of $292 \mathrm{~nm}$ using $0.1 \mathrm{~N} \mathrm{HCl}$ solution as a blank. The absorbance of Flavoxate $\mathrm{HCl}$ standard solution was also measured at a wavelength of $292 \mathrm{~nm}$. The percent of Flavoxate $\mathrm{HCl}$ released in the dissolution medium was calculated using the following formula (12).

Percentage relase of FLV HCL per tablet =

$$
=\frac{\mathrm{As}}{\mathrm{Ast}} \times \frac{\mathrm{Wst}}{\mathrm{Dst}} \times \frac{900}{1} \times \frac{100}{5} \times \frac{\mathrm{C}}{600}
$$

Where, As = absorbance of sample solution, Ast $=$ absorbance of standard solution, $\mathrm{Wst}=$ weight of working standard, Dst = dilution of standard, $1=$ number of tablets used, $600=\mathrm{mg}$ of Flavoxate $\mathrm{HCl}$ per tablet, $\mathrm{C}=\%$ age potency of standard of Flavoxate $\mathrm{HCl}$.

After $2 \mathrm{~h}$, the tablets were transferred in phosphate buffer $\mathrm{pH} 7.4$ medium previously maintained at a temperature of $37 \pm 0.5^{\circ} \mathrm{C}$. Dissolution was performed for $22 \mathrm{~h}$ with paddle speed kept at $100 \mathrm{rpm}$. Samples were drawn at $4,8,12,16$, and $24 \mathrm{~h}$, and the absorbance was noted at a wavelength of $292 \mathrm{~nm}$. The percent of Flavoxate $\mathrm{HCl}$ released was computed using Eq. 1.

\section{Stability testing}

Stability testing was carried out on two successful batches, i.e., one manufactured with HPMC and the other with Kollidon SR in accelerated conditions of temperature $\left(40 \pm 2^{\circ} \mathrm{C}\right)$ and relative humidity $(75 \pm 5 \%)$. The batches were tested for appearance, loss on drying, average weight, disintegration, in-vitro dissolution, and determination of related substance (3-methylflavone-8-carboxylic acid) at 0 , 1, 2, 3 and 6 months. TLC method was performed for determination of related substances (16). All the results are presented as the mean \pm standard deviation. Statistical analysis was carried out by using MS excel 2010.

\section{RESULTS}

Physical characteristics of Flavoxate HCI inner
core granules with HPMC K15 and Kollidon SR

Physical characteristics of inner core granules with HPMC K15 and Kollidon SR are summarized in Table 3. The appearance of all granules shows uniform white texture without any agglomeration. The

Table 3. Physical characteristics of Flavoxate $\mathrm{HCl}$ inner core granules with HPMC K15 and Kollidon SR.

\begin{tabular}{|c|c|c|c|c|c|c|}
\hline $\begin{array}{l}\text { Formulation } \\
\text { code }\end{array}$ & $\begin{array}{c}\% \text { Fines below } 60 \text { No. } \\
\text { Mean } \pm \text { SD }\end{array}$ & $\begin{array}{l}\text { Bulk density } \\
\text { Mean } \pm \text { SD }\end{array}$ & $\begin{array}{c}\text { Tapped density } \\
\text { Mean } \pm \text { SD }\end{array}$ & $\begin{array}{l}\% \text { Volume } \\
\text { decrease }\end{array}$ & $\begin{array}{l}\text { Hausner } \\
\text { Ratio }\end{array}$ & LOD Mean \pm SD \\
\hline $\mathrm{ICG}_{\mathrm{WG}-\mathrm{H}}-\mathrm{I}$ & $64 \pm 1.16$ & $0.55 \pm 0.01$ & $0.66 \pm 0.04$ & 120 & 0.83 & $2.51 \pm 0.01$ \\
\hline $\mathrm{ICG}_{\mathrm{WG}-\mathrm{K}}-\mathrm{I}$ & $78 \pm 0.95$ & $0.64 \pm 0.01$ & $0.75 \pm 0.01$ & 117.19 & 0.85 & $2.49 \pm 0.06$ \\
\hline $\mathrm{ICG}_{\mathrm{WG}-\mathrm{H}}-\mathrm{II}$ & $67 \pm 0.94$ & $0.59 \pm 0.01$ & $0.68 \pm 0.03$ & 115.25 & 0.87 & $2.68 \pm 0.01$ \\
\hline $\mathrm{ICG}_{\mathrm{WG}-\mathrm{K}}-\mathrm{II}$ & $75 \pm 0.99$ & $0.61 \pm 0.01$ & $0.74 \pm 0.01$ & 121.31 & 0.82 & $2.58 \pm 0.08$ \\
\hline $\mathrm{ICG}_{\mathrm{WG}-\mathrm{H}}-\mathrm{III}$ & $69 \pm 0.88$ & $0.60 \pm 0.01$ & $0.69 \pm 0.01$ & 115 & 0.87 & $2.59 \pm 0.01$ \\
\hline $\mathrm{ICG}_{\mathrm{WG}-\mathrm{K}}-\mathrm{III}$ & $71 \pm 0.99$ & $0.60 \pm 0.01$ & $0.71 \pm 0.01$ & 118.33 & 0.85 & $2.58 \pm 0.04$ \\
\hline $\mathrm{ICG}_{\mathrm{WG}-\mathrm{H}}-\mathrm{IV}$ & $70 \pm 0.85$ & $0.61 \pm 0.01$ & $0.71 \pm 0.03$ & 116.39 & 0.86 & $2.61 \pm 0.01$ \\
\hline $\mathrm{ICG}_{\mathrm{WG}-\mathrm{K}} \mathrm{IV}$ & $69 \pm 0.71$ & $0.65 \pm 0.01$ & $0.70 \pm 0.01$ & 107.69 & 0.93 & $2.58 \pm 0.02$ \\
\hline $\mathrm{ICG}_{\mathrm{WG}-\mathrm{H}}-\mathrm{V}$ & $67 \pm 0.63$ & $0.58 \pm 0.01$ & $0.66 \pm 0.01$ & 113.79 & 0.88 & $2.58 \pm 0.02$ \\
\hline $\mathrm{ICG}_{\mathrm{WG}-\mathrm{K}}-\mathrm{V}$ & $65 \pm 0.99$ & $0.56 \pm 0.01$ & $0.70 \pm 0.01$ & 125.00 & 0.80 & $2.52 \pm 0.04$ \\
\hline $\mathrm{ICG}_{\mathrm{WG}-\mathrm{H}}-\mathrm{VI}$ & $70 \pm 0.67$ & $0.56 \pm 0.01$ & $0.69 \pm 0.04$ & 123.21 & 0.81 & $2.52 \pm 0.01$ \\
\hline $\mathrm{ICG}_{\mathrm{WG}-\mathrm{K}}-\mathrm{VI}$ & $68 \pm 0.74$ & $0.56 \pm 0.01$ & $0.70 \pm 0.01$ & 125.00 & 0.80 & $2.56 \pm 0.05$ \\
\hline $\mathrm{ICG}_{\mathrm{WG}-\mathrm{H}}-\mathrm{VII}$ & $68 \pm 0.97$ & $0.56 \pm 0.01$ & $0.71 \pm 0.04$ & 126.79 & 0.79 & $2.55 \pm 0.01$ \\
\hline $\mathrm{ICG}_{\mathrm{WG}-\mathrm{K}}-\mathrm{VII}$ & $70 \pm 0.97$ & $0.58 \pm 0.01$ & $0.69 \pm 0.01$ & 118.97 & 0.84 & $2.49 \pm 0.02$ \\
\hline $\mathrm{ICG}_{\mathrm{WG}-\mathrm{H}}-\mathrm{VIII}$ & $69 \pm 0.82$ & $0.55 \pm 0.01$ & $0.68 \pm 0.01$ & 123.64 & 0.81 & $2.56 \pm 0.01$ \\
\hline $\mathrm{ICG}_{\mathrm{WG}-\mathrm{K}}-\mathrm{VIII}$ & $68 \pm 0.67$ & $0.58 \pm 0.01$ & $0.72 \pm 0.01$ & 124.14 & 0.81 & $2.58 \pm 0.03$ \\
\hline $\mathrm{ICG}_{\mathrm{WG}-\mathrm{H}}-\mathrm{IX}$ & $68 \pm 0.79$ & $0.56 \pm 0.01$ & $0.71 \pm 0.05$ & 126.79 & 0.79 & $2.49 \pm 0.02$ \\
\hline $\mathrm{ICG}_{\mathrm{WG}-\mathrm{K}}-\mathrm{IX}$ & $67 \pm 0.70$ & $0.49 \pm 0.01$ & $0.68 \pm 0.01$ & 138.78 & 0.72 & $2.61 \pm 0.04$ \\
\hline
\end{tabular}

*(Where WG-H and WG-K represents wet granulation with HPMC K15 and wet granulation Kollidon SR, respectively) 
sieve analysis result showed that the percentage of fine below sieve No. 60 is within acceptable limits. The percentage volume decrease was found above $100 \%$ in all cases. Similarly, Hausner ratio values showed better flow and compression properties for both types of granules. The granules prepared from HPMC K15 and Kollidon SR showed comparable bulk characteristics. All formulations have shown percent weight loss (LOD) between 2.49-2.68\%, which signifies the presence of moisture for better compression and consolidation properties of inner core granules.

\section{Thermal characterization of inner core}

As per literature, Flavoxate $\mathrm{HCl}$ has a melting point of around $232-234^{\circ} \mathrm{C}$ and the presence of a single endothermic peak at a melting point is indicative of no polymorphic transition (17). DSC thermogram (Fig. 1) of inner core granules showed a sharp endothermic peak at around $234^{\circ} \mathrm{C}-237^{\circ} \mathrm{C}$ corresponding to the melting point of the drug. Both thermograms (HPMC K15 and Kollidon SR inner core granules) displayed shallow endothermic peaks above $100^{\circ} \mathrm{C}$ which can be attributed to the loss of moisture. These results are consistent with the LOD results as presented in Table 3.

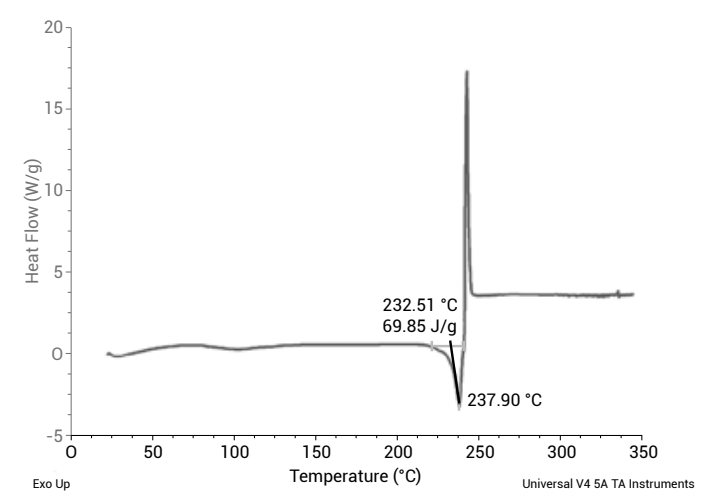

\section{FTIR analysis of inner core granules}

Figure 2 shows a comparison of the FTIR spectra of inner core granules prepared with HPMC K15 and Kollidon SR with pure drugs. FTIR spectra of the pure drug showed all the principal peaks of Flavoxate $\mathrm{HCl}$ as per literature (18). The major peaks at $1717 \mathrm{~cm}^{-1}$ and $1205 \mathrm{~cm}^{-1}$ are attributed to $\mathrm{C}=\mathrm{O}$ ester and $\mathrm{C}-\mathrm{N}$ stretching band. Moreover, the HPMC $\mathrm{K} 15$ and Kollidon SR granules spectrum showed characteristic ester $\mathrm{C}=\mathrm{O}$ and $\mathrm{C}-\mathrm{N}$ stretching bands of pure drug confirming the lack of chemical interaction between drug and polymer.

The results of content uniformity were found to be in the range of $99.1-100.12 \%$ as given in Table 4 .

\section{Characterization of inner core tablets}

The results of the physical characterization of all the batches prepared from HPMC K15 and Kollidon SR are given in Table 4. The appearance of tablets from all batches was uniform having white to off white color. The friability of all the batches was found to be in the range of $0.18 \pm 0.16 \%$ to $0.88 \pm 0.01 \%$. The hardness was found to be in the range of $5.11 \pm 0.06 \mathrm{kP}$ to $7.56 \pm 0.36 \mathrm{kP}$. The weight variation of all the tablets was within the limits, i.e., $<5 \%$.

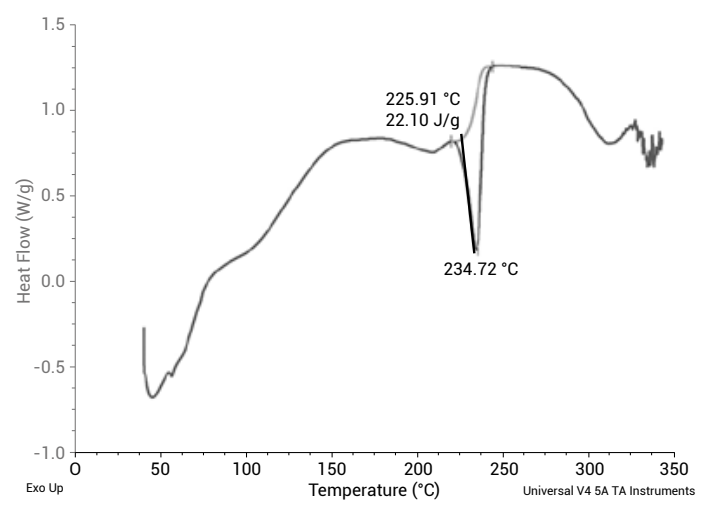

Figure 1. DSC thermogram for Flavoxate $\mathrm{HCl}$ inner core granules with HPMC K15 (A), DSC thermogram for Flavoxate $\mathrm{HCl}$ inner core granules with Kollidon (B).

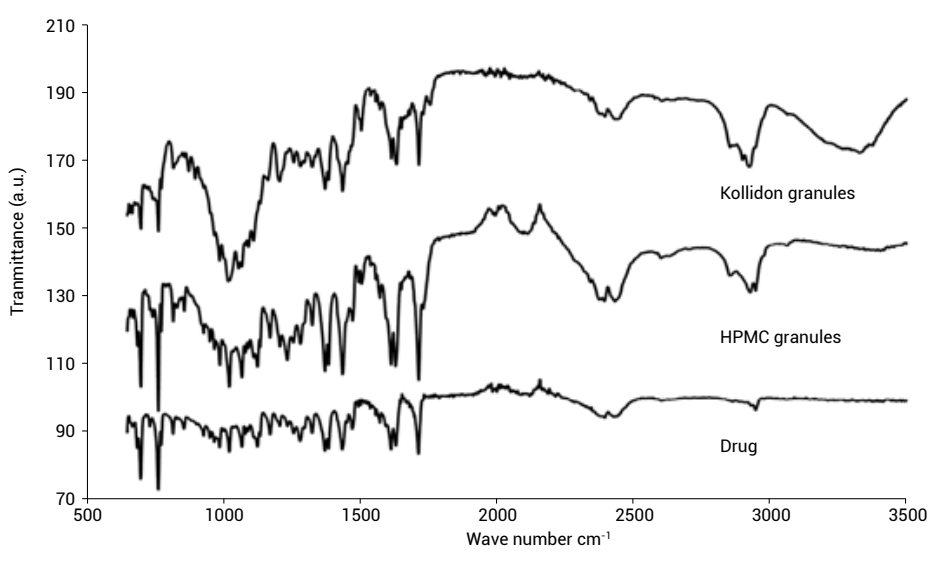

Figure 2. Comparison of ATR Spectra of HPMC K15 and Kollidon SR granules with pure drug. 
Table 4. Physical properties of Flavoxate $\mathrm{HCl}$ inner core tablet with HPMC K15.

\begin{tabular}{|c|c|c|c|c|c|c|}
\hline $\begin{array}{l}\text { Formulation } \\
\text { code }\end{array}$ & $\begin{array}{c}\text { Content uniformity } \\
\text { of blend } \\
\text { Mean } \pm \text { SD }\end{array}$ & $\begin{array}{l}\text { Diameter }(\mathrm{mm}) \\
\text { Mean } \pm \text { SD }\end{array}$ & $\begin{array}{l}\text { Friability } \\
\quad(\%)\end{array}$ & $\begin{array}{c}\text { Hardness }(\mathrm{kP}) \\
\text { Mean } \pm \mathrm{SD}\end{array}$ & $\begin{array}{c}\text { Thickness }(\mathrm{mm}) \\
\text { Mean } \pm \text { SD }\end{array}$ & $\begin{array}{c}\text { Average } \\
\text { weight } \\
\text { Mean } \pm \text { SD }\end{array}$ \\
\hline $\mathrm{ICT}_{\mathrm{WG}^{-} \mathrm{H}^{-} \mathrm{I}}$ & $99.12 \pm 0.56$ & $12.01 \pm 0.01$ & $0.83 \pm 0.13$ & $5.17 \pm 0.17$ & $5.33 \pm 0.03$ & $590 \pm 0.69$ \\
\hline $\mathrm{ICT}_{\mathrm{WG}-\mathrm{K}} \mathrm{I}$ & $99.32 \pm 0.07$ & $12.01 \pm 0.01$ & $0.88 \pm 0.01$ & $5.11 \pm 0.06$ & $5.44 \pm 0.03$ & $590 \pm 0.81$ \\
\hline $\mathrm{ICT}_{\mathrm{WG}^{-}{ }^{-}{ }^{-} \mathrm{II}}$ & $99.51 \pm 0.26$ & $12.01 \pm 0.01$ & $0.82 \pm 0.28$ & $5.71 \pm 0.22$ & $5.37 \pm 0.10$ & $610 \pm 0.78$ \\
\hline $\mathrm{ICT}_{\mathrm{WG}-\mathrm{K}}-\mathrm{II}$ & $99.43 \pm 0.03$ & $12.01 \pm 0.01$ & $00.75 \pm 0.12$ & $6.25 \pm 0.12$ & $5.42 \pm 0.02$ & $591 \pm 0.99$ \\
\hline $\mathrm{ICT}_{\mathrm{WG}^{-}{ }_{\mathrm{H}}-\mathrm{III}}$ & $100.12 \pm 0.53$ & $12.01 \pm 0.01$ & $0.75 \pm 0.33$ & $5.79 \pm 0.14$ & $5.40 \pm 0.11$ & $640 \pm 0.76$ \\
\hline $\mathrm{ICT}_{\mathrm{WG}-\mathrm{K}}-\mathrm{III}$ & $99.43 \pm 0.08$ & $12.01 \pm 0.01$ & $0.74 \pm 0.15$ & $6.13 \pm 0.05$ & $5.40 \pm 0.02$ & $593 \pm 0.94$ \\
\hline $\mathrm{ICT}_{\mathrm{WG}^{-}{ }^{-}-\mathrm{IV}}$ & $99.61 \pm 0.29$ & $12.01 \pm 0.01$ & $0.55 \pm 0.69$ & $6.06 \pm 0.32$ & $5.42 \pm 0.07$ & $650 \pm 0.91$ \\
\hline $\mathrm{ICT}_{\mathrm{WG}-\mathrm{K}}-\mathrm{IV}$ & $99.45 \pm 0.04$ & $12.01 \pm 0.01$ & $0.43 \pm 0.18$ & $7.56 \pm 0.36$ & $5.46 \pm 0.03$ & $592 \pm 0.98$ \\
\hline 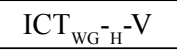 & $99.82 \pm 0.25$ & $12.01 \pm 0.01$ & $0.41 \pm 0.52$ & $6.28 \pm 0.50$ & $5.48 \pm 0.12$ & $655 \pm 0.80$ \\
\hline $\mathrm{ICT}_{\mathrm{WG}-\mathrm{K}}-\mathrm{V}$ & $99.95 \pm 0.10$ & $12.01 \pm 0.01$ & $0.18 \pm 0.16$ & $7.52 \pm 0.04$ & $5.53 \pm 0.04$ & $592 \pm 0.93$ \\
\hline $\mathrm{ICT}_{\mathrm{WG}^{-}{ }_{\mathrm{H}}-\mathrm{VI}}$ & $100.23 \pm 0.21$ & $12.01 \pm 0.01$ & $0.38 \pm 0.43$ & $6.52 \pm 0.26$ & $5.45 \pm 0.05$ & $660 \pm 0.80$ \\
\hline $\mathrm{ICT}_{\mathrm{WG}-\mathrm{K}}-\mathrm{VI}$ & $100.12 \pm 0.08$ & $12.01 \pm 0.01$ & $0.21 \pm 0.19$ & $7.55 \pm 0.10$ & $5.42 \pm 0.02$ & $591 \pm 0.95$ \\
\hline 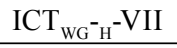 & $99.88 \pm 0.25$ & $12.01 \pm 0.01$ & $0.34 \pm 0.22$ & $7.09 \pm 0.26$ & $5.47 \pm 0.13$ & $650 \pm 0.92$ \\
\hline $\mathrm{ICT}_{\mathrm{WG}-\mathrm{K}}-\mathrm{VII}$ & $99.38 \pm 0.10$ & $12.01 \pm 0.01$ & $0.24 \pm 0.36$ & $7.34 \pm 0.63$ & $5.41 \pm 0.03$ & $592 \pm 0.81$ \\
\hline $\mathrm{ICT}_{\mathrm{WG}^{-} \mathrm{H}^{-}-\mathrm{VIII}}$ & $99.67 \pm 0.30$ & $12.01 \pm 0.01$ & $0.27 \pm 0.29$ & $7.18 \pm 0.27$ & $5.48 \pm 0.12$ & $655 \pm 0.88$ \\
\hline $\mathrm{ICT}_{\mathrm{WG}-\mathrm{K}}-\mathrm{VIII}$ & $99.58 \pm 0.05$ & $12.01 \pm 0.01$ & $0.22 \pm 0.26$ & $7.56 \pm 0.10$ & $5.44 \pm 0.03$ & $592 \pm 0.95$ \\
\hline $\mathrm{ICT}_{\mathrm{WG}^{-}{ }_{\mathrm{H}}-\mathrm{IX}}$ & $99.19 \pm 0.18$ & $12.01 \pm 0.01$ & $0.25 \pm 0.47$ & $7.44 \pm 0.46$ & $5.50 \pm 0.12$ & $660 \pm 0.78$ \\
\hline $\mathrm{ICT}_{\mathrm{WG}-\mathrm{K}}-\mathrm{IX}$ & $99.69 \pm 0.03$ & $12.01 \pm 0.01$ & $0.23 \pm 0.27$ & $7.73 \pm 0.16$ & $5.44 \pm 0.03$ & $592 \pm 0.73$ \\
\hline
\end{tabular}

Table 5. Physical properties Flavoxate $\mathrm{HCl}$ outer core granules.

\begin{tabular}{|c|c|c|c|c|c|}
\hline $\begin{array}{c}\text { Formulation } \\
\text { code }\end{array}$ & $\begin{array}{c}\text { Flavoxate HCl content } \\
\text { Mean } \pm \text { SD }\end{array}$ & $\begin{array}{c}\text { Fines below 60 No. } \\
\text { Mean } \pm \text { SD }\end{array}$ & $\begin{array}{c}\text { Bulk Density } \\
\text { Mean } \pm \text { SD }\end{array}$ & $\begin{array}{c}\text { Tapped density } \\
\text { Mean } \pm \text { SD }\end{array}$ & $\begin{array}{c}\text { Loss on Drying } \\
\text { Mean } \pm \text { SD }\end{array}$ \\
\hline $\mathrm{OCG}_{\mathrm{WG}}-\mathrm{I}$ & $99.10 \pm 0.51$ & $54 \pm 0.01$ & $0.54 \pm 0.02$ & $0.71 \pm 0.02$ & $2.61 \pm 0.01$ \\
\hline $\mathrm{OCG}_{\mathrm{WG}}-\mathrm{II}$ & $100.12 \pm 0.79$ & $61 \pm 0.02$ & $0.56 \pm 0.03$ & $0.69 \pm 0.06$ & $2.55 \pm 0.01$ \\
\hline $\mathrm{OCG}_{\mathrm{WG}}-\mathrm{III}$ & $99.25 \pm 0.60$ & $65 \pm 0.01$ & $0.59 \pm 0.02$ & $0.65 \pm 0.06$ & $2.53 \pm 0.02$ \\
\hline
\end{tabular}

\section{Physical characteristics of outer core granules}

Three different outer core immediate release batches of Flavoxate $\mathrm{HCl}$ were prepared and results of physical properties are presented in Table 5. Sieve Analysis of all batches showed the percentage of fine below sieve No. 60 within $54 \pm 0.01 \%$ to $65 \pm 0.01 \%$. The LOD values ranged from
$2.53 \pm 0.02 \%$ to $2.61 \pm 0.01 \%$ for three outer core batches.

\section{Thermal analysis of outer core granules}

DSC thermogram of outer core granules (Fig. 3) shows a short endothermic peak at $243^{\circ} \mathrm{C}$ which represents the melting of the drug. There is a shallow

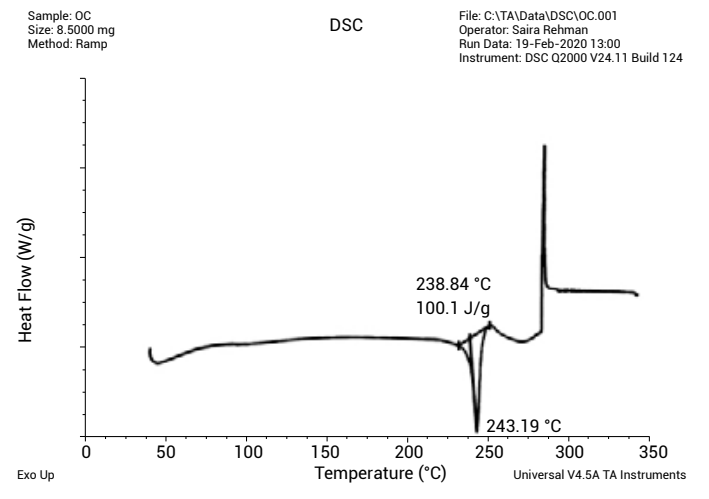

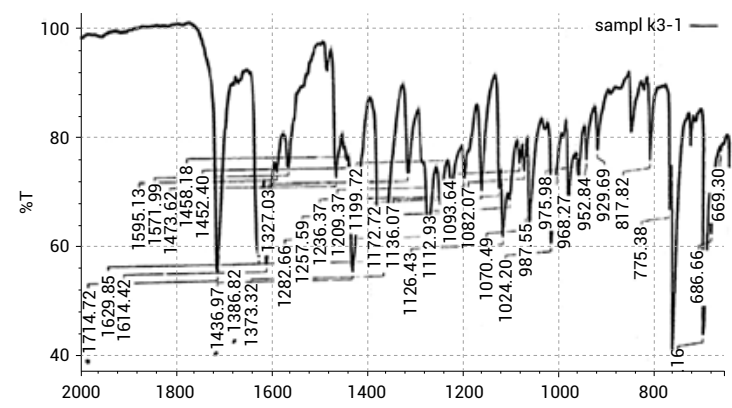

Figure 3. DSC thermogram and ATR for Flavoxate $\mathrm{HCl}$ outer core granules. 
Table 6. Physical properties of Flavoxate $\mathrm{HCl}$ double core tablets with HPMC K15.

\begin{tabular}{|c|c|c|c|c|c|}
\hline $\begin{array}{l}\text { Formulation } \\
\text { Code }\end{array}$ & $\begin{array}{c}\text { Diameter }(\mathrm{mm}) \\
\text { Mean } \pm \text { SD }\end{array}$ & $\begin{array}{c}\text { Hardness }(\mathrm{kP}) \\
\text { Mean } \pm \text { SD }\end{array}$ & $\begin{array}{c}\text { Thickness }(\mathrm{mm}) \\
\text { Mean } \pm \text { SD }\end{array}$ & $\begin{array}{l}\text { Average weight (mg) } \\
\text { Mean } \pm \text { SD }\end{array}$ & $\begin{array}{l}\text { Disintegration time }(\mathrm{min}) \\
\text { Mean } \pm \mathrm{SD}\end{array}$ \\
\hline $\mathrm{DCT}_{\mathrm{H}-\mathrm{WG}}-\mathrm{I}$ & $16.01 \pm 0.01$ & $11.20 \pm 0.24$ & $6.32 \pm 0.04$ & $990.71 \pm 0.42$ & $10.50 \pm 0.28$ \\
\hline $\mathrm{DCT}_{\mathrm{K}-\mathrm{WG}}-\mathrm{I}$ & $16.01 \pm 0.01$ & $10.14 \pm 0.08$ & $6.39 \pm 0.04$ & $990.21 \pm 0.35$ & $10.40 \pm 0.35$ \\
\hline $\mathrm{DCT}_{\mathrm{H}-\mathrm{WG}}-\mathrm{II}$ & $16.01 \pm 0.01$ & $10.85 \pm 0.09$ & $6.35 \pm 0.02$ & $1011.59 \pm 0.69$ & $11.00 \pm 0.39$ \\
\hline $\mathrm{DCT}_{\mathrm{K}-\mathrm{WG}}-\mathrm{II}$ & $16.01 \pm 0.01$ & $10.82 \pm 0.05$ & $6.39 \pm 0.04$ & $992.21 \pm 0.91$ & $10.50 \pm 0.21$ \\
\hline $\mathrm{DCT}_{\mathrm{H}-\mathrm{WG}}-\mathrm{III}$ & $16.01 \pm 0.01$ & $11.80 \pm 0.09$ & $6.38 \pm 0.03$ & $1040.51 \pm 0.69$ & $11.20 \pm 0.43$ \\
\hline $\mathrm{DCT}_{\mathrm{K}-\mathrm{WG}}-\mathrm{III}$ & $16.01 \pm 0.01$ & $10.81 \pm 0.05$ & $6.39 \pm 0.04$ & $991.41 \pm 0.35$ & $10.20 \pm 0.17$ \\
\hline $\mathrm{DCT}_{\mathrm{H}-\mathrm{wG}}-\mathrm{IV}$ & $16.01 \pm 0.01$ & $11.66 \pm 0.19$ & $6.42 \pm 0.01$ & $1051.81 \pm 0.82$ & $10.30 \pm 0.25$ \\
\hline $\mathrm{DCT}_{\mathrm{K}-\mathrm{WG}}-\mathrm{IV}$ & $16.01 \pm 0.01$ & $11.36 \pm 0.09$ & $6.40 \pm 0.03$ & $990.26 \pm 0.73$ & $10.30 \pm 0.25$ \\
\hline $\mathrm{DCT}_{\mathrm{H}-\mathrm{WG}}-\mathrm{V}$ & $16.02 \pm 0.02$ & $12.14 \pm 0.13$ & $6.31 \pm 0.04$ & $1055.09 \pm 0.74$ & $10.40 \pm 0.49$ \\
\hline $\mathrm{DCT}_{\mathrm{K}-\mathrm{WG}}-\mathrm{V}$ & $16.02 \pm 0.04$ & $11.57 \pm 0.08$ & $6.40 \pm 0.02$ & $993.34 \pm 0.85$ & $10.50 \pm 0.23$ \\
\hline $\mathrm{DCT}_{\mathrm{H}-\mathrm{WG}}-\mathrm{VI}$ & $16.01 \pm 0.01$ & $12.48 \pm 0.11$ & $6.42 \pm 0.01$ & $1060.74 \pm 0.91$ & $10.50 \pm 0.38$ \\
\hline $\mathrm{DCT}_{\mathrm{K}-\mathrm{WG}}-\mathrm{VI}$ & $16.01 \pm 0.01$ & $11.63 \pm 0.17$ & $6.42 \pm 0.01$ & $991.23 \pm 0.15$ & $11.50 \pm 0.18$ \\
\hline $\mathrm{DCT}_{\mathrm{H}-\mathrm{WG}} \mathrm{VII}$ & $16.01 \pm 0.01$ & $12.41 \pm 0.41$ & $6.45 \pm 0.03$ & $1054.90 \pm 0.84$ & $11.10 \pm 0.55$ \\
\hline $\mathrm{DCT}_{\mathrm{K}-\mathrm{WG}} \mathrm{VII}$ & $16.01 \pm 0.01$ & $11.59 \pm 0.03$ & $6.39 \pm 0.04$ & $991.35 \pm 0.65$ & $11.00 \pm 0.26$ \\
\hline $\mathrm{DCT}_{\mathrm{H}-\mathrm{WG}}-\mathrm{VIII}$ & $16.01 \pm 0.01$ & $12.74 \pm 0.03$ & $6.47 \pm 0.02$ & $1059.47 \pm 0.56$ & $11.00 \pm 0.47$ \\
\hline $\mathrm{DCT}_{\mathrm{K}-\mathrm{WG}}-\mathrm{VIII}$ & $16.01 \pm 0.01$ & $11.62 \pm 0.31$ & $6.44 \pm 0.02$ & $992.25 \pm 0.82$ & $11.30 \pm 0.41$ \\
\hline $\mathrm{DCT}_{\mathrm{H}-\mathrm{WG}}-\mathrm{IX}$ & $16.01 \pm 0.01$ & $12.85 \pm 0.08$ & $6.48 \pm 0.02$ & $1060.94 \pm 0.92$ & $11.10 \pm 0.53$ \\
\hline $\mathrm{DCT}_{\mathrm{K}-\mathrm{WG}}-\mathrm{IX}$ & $16.01 \pm 0.01$ & $11.65 \pm 0.17$ & $6.46 \pm 0.03$ & $993.59 \pm 0.76$ & $11.10 \pm 0.27$ \\
\hline
\end{tabular}

endotherm at $100^{\circ} \mathrm{C}$ representing moisture loss. These results agree with the DSC results of inner core granules.

\section{FTIR analysis and content uniformity of Flavoxate HCl outer core granules}

FTIR spectrum of outer granules (Fig. 3) displayed all the principal peaks of drugs which indicate that no interaction occurred between drug and polymer. Uniformity of content was performed for all the three Flavoxate $\mathrm{HCl}$ outer core granules by a validated HPLC method. The results indicated that peak area of standard preparation corresponds to that of sample preparation. The results of the content uniformity test (Table 5) were found to be in between $99.10 \pm 0.51$ to $100.12 \pm 0.79 \%$.

\section{Physical characteristics of Flavoxate $\mathrm{HCl}$ double core tablets with HPMC K15/Kollidon SR}

Physical characteristics of all the double core Flavoxate $\mathrm{HCl}$ batches are given in Table 6. The appearance of double core Flavoxate $\mathrm{HCl}$ tablets was from white to off white plain tablets. The hardness was in the range of $10.14 \pm 0.08 \mathrm{kP}$ to $12.85 \pm 0.08 \mathrm{kP}$. The average tablet weight was observed to be in the range of $990.21 \pm 0.35 \mathrm{mg}$ to $1060.94 \pm 0.92 \mathrm{mg}$, lying within the official BP limits (12). The disintegration time was observed to be in the range of $10.20 \pm 0.17 \mathrm{~min}$ to $11.50 \pm 0.18 \mathrm{~min}$.

\section{Dissolution studies for Flavoxate $\mathrm{HCl}$ double core tablets with HPMC K15 and Kollidon SR}

The dissolution of Flavoxate $\mathrm{HCl}$ double core tablets with HPMC K15 and Kollidon SR was performed in dissolution media of $0.1 \mathrm{~N} \mathrm{HCl}(\mathrm{pH} 1.2)$ and phosphate buffer ( $\mathrm{pH} 7.4)$ and the results are given in Table 7 and 8 and Figure 4. Batch $\mathrm{DCT}_{\mathrm{H}-\mathrm{WG}}-\mathrm{I}$ failed to sustain the release of the drug for $2 \mathrm{~h}$ in $0.1 \mathrm{~N}$ $\mathrm{HCl}$. The in-vitro release data of the drug was fitted to various release kinetic models. Drug release data of swellable matrix system $\mathrm{DCT}_{\mathrm{H}^{-} \mathrm{WG}-\mathrm{I} \text { was }}$ best fitted according to Korsmeyer-Peppas model, while the release data for the other eight batches

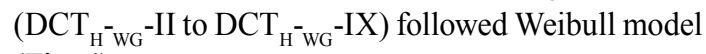
(Fig. 4).

All the double core batches with Kollidon SR released the drug at various time intervals in a $7.4 \mathrm{pH}$ phosphate buffer. The in-vitro release data were fitted to various kinetic models and all the batches (DCT $\bar{K}^{-}{ }^{-}-\mathrm{IV}$ to $\mathrm{DCT}_{\mathrm{K}^{-}{ }_{\mathrm{WG}}}-\mathrm{IX}$ ) were observed to follow the Weibull model (Fig. 4). 
Table 7. Percentage release of Flavoxate $\mathrm{HCl}$ double core tablets with HPMC K15.

\begin{tabular}{|c|c|c|c|c|c|c|c|c|}
\hline \multirow{2}{*}{$\begin{array}{c}\text { Formulation } \\
\text { Code }\end{array}$} & \multicolumn{6}{|c|}{$\begin{array}{c}\text { Dissolution profile of Flavoxate HCl } \\
\text { tablets (Sustained release) in } 0.1 \mathrm{~N} \mathrm{HCl}\end{array}$} & \multicolumn{5}{|c|}{$\begin{array}{c}\text { Dissolution profile of Flavoxate HCl tablets(Sustained } \\
\text { release) in Phosphate Buffer 7.4 }\end{array}$} \\
\cline { 2 - 10 } & $0.5 \mathrm{hr}$ & $1.0 \mathrm{hr}$ & $2.0 \mathrm{hr}$ & $4 \mathrm{hr}$ & $8 \mathrm{hr}$ & $12 \mathrm{hr}$ & $16 \mathrm{hr}$ & $24 \mathrm{hr}$ \\
\hline $\mathrm{DCT}_{\mathrm{H}-\mathrm{WG}}$-I & 13.28 & 50.55 & 99.19 & - & - & - & - & - \\
\hline $\mathrm{DCT}_{\mathrm{H}-\mathrm{WG}}-\mathrm{II}$ & 14.08 & 40.84 & 71.11 & 98.48 & - & - & - & - \\
\hline $\mathrm{DCT}_{\mathrm{H}-\mathrm{WG}}-\mathrm{III}$ & 13.15 & 30.37 & 45.16 & 85.31 & 97.62 & - & - & - \\
\hline $\mathrm{DCT}_{\mathrm{H}-\mathrm{WG}}-\mathrm{IV}$ & 14.01 & 28.31 & 38.43 & 75.41 & 89.42 & 99.29 & - & - \\
\hline $\mathrm{DCT}_{\mathrm{H}-\mathrm{WG}}-\mathrm{V}$ & 13.12 & 24.21 & 31.25 & 65.47 & 77.29 & 98.59 & - & - \\
\hline $\mathrm{DCT}_{\mathrm{H}-\mathrm{WG}}-\mathrm{VI}$ & 14.22 & 22.56 & 27.23 & 56.21 & 69.25 & 83.29 & 98.95 & - \\
\hline $\mathrm{DCT}_{\mathrm{H}-\mathrm{WG}}-\mathrm{VII}$ & 14.09 & 20.32 & 24.35 & 34.21 & 52.23 & 67.25 & 87.65 & 97.26 \\
\hline $\mathrm{DCT}_{\mathrm{H}-\mathrm{WG}} \mathrm{VIII}$ & 13.97 & 16.25 & 19.59 & 29.36 & 39.29 & 57.36 & 78.23 & 83.99 \\
\hline $\mathrm{DCT}_{\mathrm{H}-\mathrm{WG}}-\mathrm{IX}$ & 14.05 & 17.21 & 20.35 & 33.26 & 48.47 & 62.53 & 81.39 & 98.51 \\
\hline
\end{tabular}

Table 8. Percentage release of Flavoxate $\mathrm{HCl}$ double core tablets with Kollidon SR.

\begin{tabular}{|c|c|c|c|c|c|c|c|c|}
\hline \multirow{2}{*}{$\begin{array}{c}\text { Formulation } \\
\text { Code }\end{array}$} & \multicolumn{2}{|c|}{$\begin{array}{c}\text { Dissolution profile of Flavoxate } \mathrm{HCl} \\
\text { tablets (Sustained release) } \\
\text { in 0.1 N HCl\% released }\end{array}$} & \multicolumn{5}{|c|}{$\begin{array}{c}\text { Dissolution profile of Flavoxate HCl tablets } \\
\text { (Sustained release) in } \\
\text { Phosphate Buffer 7.4\% released }\end{array}$} \\
\cline { 2 - 10 } & $0.5 \mathrm{hr}$ & $1.0 \mathrm{hr}$ & $2.0 \mathrm{hr}$ & $4 \mathrm{hr}$ & $8 \mathrm{hr}$ & $12 \mathrm{hr}$ & $16 \mathrm{hr}$ & $24 \mathrm{hr}$ \\
\hline $\mathrm{DCT}_{\mathrm{K}-\mathrm{WG}}$-I & 13.2 & 36.55 & 69.19 & 97.48 & - & - & - & - \\
\hline $\mathrm{DCT}_{\mathrm{K}-\mathrm{WG}}$-II & 13.1 & 32.31 & 61.19 & 98.48 & - & - & - & - \\
\hline $\mathrm{DCT}_{\mathrm{K}-\mathrm{WG}}-\mathrm{III}$ & 14.0 & 30.37 & 52.96 & 82.11 & 97.62 & - & - & - \\
\hline $\mathrm{DCT}_{\mathrm{K}-\mathrm{WG}}-\mathrm{IV}$ & 14.1 & 25.31 & 32.43 & 70.41 & 80.02 & 98.79 & - & - \\
\hline $\mathrm{DCT}_{\mathrm{K}-\mathrm{WG}}-\mathrm{V}$ & 13.1 & 22.21 & 26.19 & 55.77 & 67.29 & 87.59 & 99.25 & - \\
\hline $\mathrm{DCT}_{\mathrm{K}-\mathrm{WG}}-\mathrm{VI}$ & 14.1 & 21.06 & 25.95 & 52.35 & 65.76 & 83.29 & 99.05 & - \\
\hline $\mathrm{DCT}_{\mathrm{K}-\mathrm{WG}}-\mathrm{VII}$ & 13.29 & 18.32 & 22.18 & 34.21 & 48.23 & 59.15 & 87.26 & 96.23 \\
\hline $\mathrm{DCT}_{\mathrm{K}-\mathrm{WG}}-\mathrm{VIII}$ & 13.97 & 16.25 & 17.29 & 25.95 & 42.73 & 54.26 & 72.36 & 82.99 \\
\hline $\mathrm{DCT}_{\mathrm{K}-\mathrm{WG}}-\mathrm{IX}$ & 14.15 & 17.42 & 20.87 & 32.17 & 45.77 & 58.29 & 78.36 & 98.51 \\
\hline
\end{tabular}
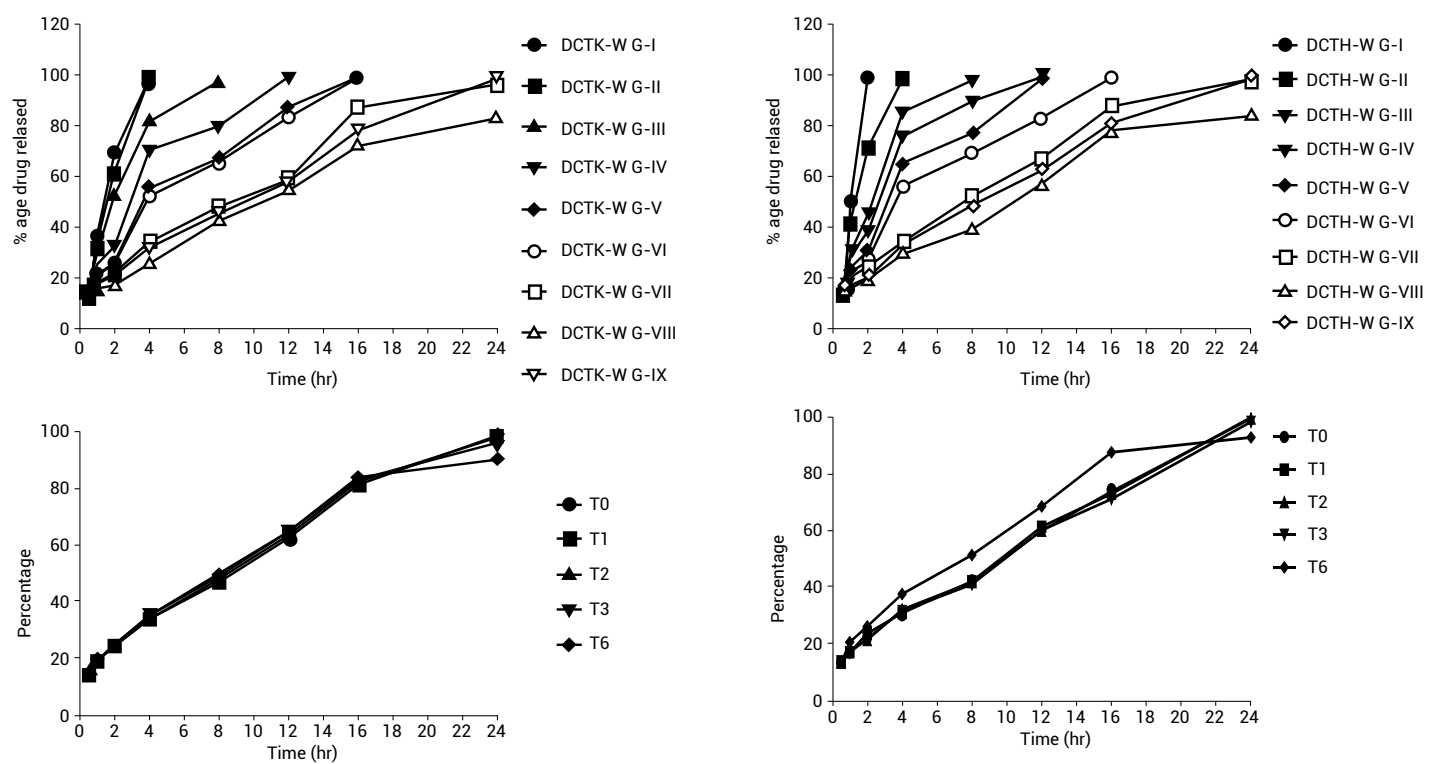

Figure 4. A) Percentage drug release versus time for swellable Flavoxate $\mathrm{HCl}$ double core tablets with Kollidon SR B) Percentage drug release versus time for swellable double core Flavoxate $\mathrm{HCl}$ tablet with HPMC K15 C) Percentage drug release versus time for swellable Flavoxate $\mathrm{HCl}$ double core tablets prepared with Kollidon SR D) Percentage drug release versus time for swellable Flavoxate $\mathrm{HCl}$ double core tablets with HPMC K15. 
Table 9. Stability studies of batches DCT $\mathrm{H}_{\mathrm{WG}}-\mathrm{IX}$ and $\mathrm{DCT}_{\mathrm{K}-\mathrm{WG}}-\mathrm{IX}$ prepared with HPMC K15 and Kollidon SR.

\begin{tabular}{|c|c|c|c|c|c|c|c|c|c|c|}
\hline \multirow{2}{*}{ Test } & \multirow{2}{*}{ Initial } & \multicolumn{3}{|c|}{$40 \pm 2^{\circ} \mathrm{C} / \mathrm{RH} 75 \pm 5 \%$} & \multirow{2}{*}{ Initial } & \multicolumn{3}{|c|}{$40 \pm 2^{\circ} \mathrm{C} / \mathrm{RH} 75 \pm 5 \%$} \\
\cline { 3 - 8 } & & 1 & 2 & 3 & 6 & & 1 & 2 & 3 & 6 \\
\hline $\begin{array}{c}\text { Dissolution } \\
0.5 \mathrm{~h}\end{array}$ & 13.78 & 13.19 & 13.75 & 14.54 & 14.13 & 10.30 & 10.50 & 10.5 & 12.50 & 17.0 \\
\hline $1 \mathrm{~h}$ & 18.17 & 18.83 & 19.51 & 19.77 & 19.91 & 13.78 & 13.61 & 13.92 & 14.13 & 13.50 \\
\hline $2 \mathrm{~h}$ & 23.32 & 24.16 & 24.23 & 24.97 & 25.06 & 17.32 & 17.31 & 17.89 & 17.21 & 20.85 \\
\hline $4 \mathrm{~h}$ & 33.15 & 33.57 & 33.61 & 34.18 & 35.42 & 22.33 & 23.95 & 21.64 & 22.24 & 26.47 \\
\hline $8 \mathrm{~h}$ & 47.26 & 47.87 & 48.21 & 49.09 & 49.36 & 30.36 & 31.09 & 32.39 & 31.87 & 37.86 \\
\hline $12 \mathrm{~h}$ & 63.24 & 63.46 & 63.88 & 64.39 & 64.46 & 42.69 & 42.37 & 42.08 & 41.25 & 51.64 \\
\hline $16 \mathrm{~h}$ & 80.33 & 80.81 & 81.97 & 82.67 & 83.37 & 61.33 & 61.64 & 60.21 & 60.39 & 68.81 \\
\hline $24 \mathrm{~h}$ & 100.4 & 99.76 & 99.86 & 89.46 & 90.19 & 74.86 & 73.23 & 74.01 & 71.37 & 87.89 \\
\hline $\begin{array}{c}\text { Assay by HPLC } \\
\begin{array}{c}\text { Each tablet contains } \\
\text { Flavoxate HCl (600 mg) }\end{array}\end{array}$ & 101.82 & 100.10 & 100.55 & 99.78 & 95.42 & 101.57 & 101.69 & 100.15 & 98.86 & 95.05 \\
\hline
\end{tabular}

\section{Stability of selected double core formulations}

From the swellable matrix tablets, two tablets, i.e., $\mathrm{DCT}_{\mathrm{H}-\mathrm{WG}}-\mathrm{IX}$, prepared with HPMC K15 and $\mathrm{DCT}_{\mathrm{K}-\mathrm{WG}}$-IX, prepared with kollidon SR were selected based on in-vitro release data to perform accelerated stability studies. The two above-stated batches gave desirable release at the time points set for sampling. The stability studies were carried out as per ICH guidelines (16). The drug release of stability samples was comparable to that of the initial samples. The results of the stability studies are given in Table 9.

\section{DISCUSSION}

To improve patient compliance, differential release tablets are one of the important innovations in the delivery of drugs where multiple dosing is required. Dual-core tablets serve the purpose quite well, where outer layer release loading dose to achieve effective plasma concentration and inner sustained-release core gives plasma concentration over a prolonged period. In this study, dual-core tablets of Flavoxate $\mathrm{HCl}$ as a model drug consisting of an inner sustained-release core and outer immediate-release layer were prepared. The granules of the inner core were formulated with HPMC K15 and Kollidon SR which were apparently white in color, without any agglomeration. It was observed that the bulk and flow properties of inner core granules were dependent on the ratio of both polymers. Batches VII, VIII, and IX showed good flow and compressional properties where higher concentrations of polymers were used. Another important requirement was the lack of chemical interaction among excipients. In our studies, FTIR data exhibited that there was no such interaction. DSC data confirmed the thermal stability of the drug. Inner core granules prepared from both HPMC K15 and Kollidon were subjected to compression into inner core tablets having optimum physical characteristics.

Outer core granules containing Flavoxate HCI were formulated by wet granulation technique using Povidone K30 as a binder. Micromeritic and compressional properties of these granules play a vital role as this layer is compressed on the inner core tablets. After performing the preliminary micromeritics studies, batch $\mathrm{OCG}_{\mathrm{WG}}$-II containing a moderate amount of binder was found to be the most appropriate one for double-core compression. Similarly, FTIR analysis of the double core tablets confirmed the absence of any interactions between excipients and drug. All the physical parameters of double core tablets compressed by using $\mathrm{OCG}_{\mathrm{WG}}$-II, i.e., content uniformity, diameter, thickness, weight variation, and hardness were observed to be within the limits given in BP (12). All compressed double core tablets were then subjected to dissolution analysis at $\mathrm{pH} 1.2$ initially. Batch $\mathrm{DCT}_{\mathrm{H}-\mathrm{WG}}-\mathrm{I}$ released the entire drug at $\mathrm{pH} 1.2$, whereas all other formulations sustained the release of the drug for more than $2 \mathrm{~h}$. Batches DCT ${ }_{\mathrm{H}-\mathrm{WG}}$-II and $\mathrm{DCT}_{\mathrm{H}-\mathrm{WG}}$-III showed sustained drug release for $4 \mathrm{~h}$ and $8 \mathrm{~h}$, respectively. While batches $\mathrm{DCT}_{\mathrm{H}-\mathrm{WG}}-\mathrm{IV}$ and $\mathrm{DCT}_{\mathrm{H}-\mathrm{WG}}-\mathrm{V}$ exhibited sustained drug release for up to $12 \mathrm{~h}$. Though the batch DCT $_{\mathrm{H}-\mathrm{WG}}-\mathrm{VII}$ could sustain the drug release for $24 \mathrm{~h}$ yet showed higher release at $16 \mathrm{~h}$ than the stipulated release limits for sustained release dosage form (20). $\mathrm{DCT}_{\mathrm{H}-\mathrm{WG}}-\mathrm{VIII}$ sustained the release of the drug for $24 \mathrm{~h}$, but the release at $24 \mathrm{~h}$ was $83.99 \%$, which was lesser than the set limit, i.e., should not 
be lesser than $85 \%$ at this time interval. The double compressed tablet batch $\mathrm{DCT}_{\mathrm{H}-\mathrm{WG}}$-IX effectively complied with all the parameters set for the sustained release formulations at various time intervals till $24 \mathrm{~h}$. The best-fitted release kinetic model was selected based on the coefficient of regression $\mathrm{R}^{2}$. $\mathrm{DCT}_{\mathrm{H}-\mathrm{WG}}-\mathrm{II}$ to $\mathrm{DCT}_{\mathrm{H}-\mathrm{WG}}$-IX was best described by the Weibull model. Batches prepared with Kollidon SR inner core showed different release profiles. Tablet batch $\mathrm{DCT}_{\mathrm{K}-\mathrm{WG}}-\mathrm{I}$ and $\mathrm{DCT}_{\mathrm{K}-\mathrm{WG}}$-II could sustain the release of the drug only for $4 \mathrm{~h}$, while batches $\mathrm{DCT}_{\mathrm{K}-\mathrm{WG}}-\mathrm{III}$ and $\mathrm{DCT}_{\mathrm{K}-\mathrm{WG}}-\mathrm{IV}$ could sustain the release of the drug for 8 and $12 \mathrm{~h}$, respectively. Formulation $\mathrm{DCT}_{\mathrm{K}-\mathrm{WG}}-\mathrm{V}$ and $\mathrm{DCTK}_{-\mathrm{WG}}-\mathrm{VI}$ sustained the drug release till $16 \mathrm{~h}$. Among all, batch $\mathrm{DCT}_{\mathrm{K}-\mathrm{WG}}$-VIII showed the lesser release at $12 \mathrm{~h}$ and the release was $82.99 \%$ which is less than the preset limit $(85 \%)$. The release profile of the batch $\mathrm{DCT}_{\mathrm{K}-\mathrm{WG}}$-IX complied with all the parameters set at various time intervals. The release kinetics for all the batches prepared with Kollidon SR inner core was best described by the Weibull model.

Although the HPMC K15 batch DCT ${ }_{\mathrm{H}-\mathrm{WG}}-\mathrm{IX}$ seemed appropriate among all the formulations in terms of the desired characteristics for a sustained release dosage form, however, the weight of the double core tablet was $1060 \mathrm{mg}$. Furthermore, the release of the drug over $24 \mathrm{~h}$ for HPMC K15 based batch $\mathrm{DCT}_{\mathrm{H}-\mathrm{WG}}$-IX was higher than the set limits for dissolution. Whereas $\mathrm{DCT}_{\mathrm{K}-\mathrm{WG}}$-IX formulated with Kollidon SR exhibits a better release profile over $24 \mathrm{~h}$ along with appropriate tablet weight.

The stability studies for the two double core tablet formulations, one manufactured with HPMC $\mathrm{K} 15$ inner core, $\mathrm{DCT}_{\mathrm{H}-\mathrm{WG}}-\mathrm{IX}$, and the other prepared with Kollidon SR inner core, $\mathrm{DCT}_{\mathrm{K}-\mathrm{WG}}$-IX were selected for the accelerated stability studies performed at $40^{\circ} \mathrm{C}$ temperature and $75 \% \mathrm{RH}$, as per ICH guidelines. The tablet batches were blister packed using opaque white PVC with 25-micron aluminum foil and were placed in the stability chamber (HPP, Memmert, Germany). Testing was carried out at T0, T1, T2, T3, and T6. The parameters studied were physical appearance, moisture content, average weight, and drug content. The findings showed satisfactory results at $\mathrm{T} 0$, T1, T2, T3 time frames, but results at T6 showed a significant increase in the moisture content of both the batches. Furthermore, the assay of both tablet batches manufactured with HPMC K15 and Kollidon SR was $95.75 \%$ and $95.07 \%$, respectively, which was within the limit for the release (NLT 95\%). Impurities were found to be remarkably increased at the T6 stage in stability studies. The average weight of the tablet remained within the specified limits. The release profile of the selected formulation was similar and followed the same kinetics model, i.e., Weibull, as discussed above in the characterization of double core tablets with HPMC K15 and Kollidon SR.

\section{CONCLUSION}

In this study, double core tablets of Flavoxate $\mathrm{HCl}$ containing sustained-release inner core and immediate release outer layer were prepared and characterized. The drug release from the inner core was sustained for a prolonged period using swellable polymers, i.e., HPMC K15 and Kollidon SR. It has been shown that a simple and reproducible compression method can be applied to prepare double core tablets having desired release characteristics of the drug.

\section{Acknowledgments}

The authors would like to acknowledge the University of Sargodha for providing support to this project.

\section{Conflict of interest}

The authors declare no conflicts of interest.

\section{REFERENCES}

1. Grassi M., Lapasn R., Pricl S.: Chem. Eng. Commun.169, 79 (1998).

2. Colombo P., Bettini R., Santi P., Peppas N.A.: Pharm. Sci. Technol. Today 3, 6 (2000).

3. Bravo S.A., Lamas M.C., Salomon C.: Pharm. Dev. Technol. 9, 1 (2004).

4. Ebube N.K., Jones A.B.: Int. J. Pharm. 272, 19 (2004).

5. https://www.pdr.net/drug-summary/FlavoxateHydrochloride-flavoxate-hydrochloride3592.116\#topPage, (accessed on 09.06.2020).

6. Bertoli M., Conti F., Conti M., Cova A., Setnikar I.: Pharmacol. Res. Commun. 8, 4 (1976).

7. Kasperek R., Zimmer L., Zun M., Dwornicka D., Wojciechowska K., et al.: Curr. Issues Pharm. Medical Sci. 29, 2 (2016).

8. Mandal U., Gowda V., Ghosh A., Selvan S., Solomon S., et al.:Yakugaku Zasshi 127, 8 (2007).

9. Qazi F., Shoaib M.H., Yousuf R.I., Qazi T.M., Mehmood Z.A., Hasan S.M.F.: Pak. J. Pharm. Sci. 26, 4 (2013). 
10. Avbunudiogba J.A.: Niger. J. Pharm. Appl. Sci. Res. 8, 107 (2019).

11. Agrawal P., Banjare T., Gupta S., Bhandarkar A., Sahu H., et al.: Res. J. Pharm. Technol. 11, 7 (2018).

12. British Pharmacopoeia, 2016.

13. Varma K.: Res. Rev. J. Chem. 5 (2016).

14. Njega E.K., Maru S.M., Tirop L.J.: Afr. J. Pharm. Sci. 21, 1 (2018).

15. Ochoa L., Igartua M., Hernandez R., Gascon A., Pedraz J.L.: J. Pharm. Pharm. Sci. 8, 2 (2005).
16. https://ich.org/page/quality-guidelines

17. Attia A.K., Mohamed G.G., Ahmed H.E.: J. Therm. Anal. Calorim. 131, 2 (2018).

18. Li C. L., Martini L.G., Ford J.L., Roberts M.: J. Pharm. Pharmacol. 57, 533 (2005).

19. Akter M., Banik S., Hossain M.S.: J. Appl. Pharm. Sci. 2, 188 (2012).

20. Alhalmi A., Altowairi M., Saeed O., Alzubaidi N., Almoiliqy M., Abdulmalik.: World J. Pharm. Pharm. Sci. 7, 6 (2018). 